\title{
High-pressure melting curve of hydrogen
}

\author{
Sergio M. Davis, ${ }^{1, \text { a) }}$ Anatoly B. Belonoshko, ${ }^{1,2}$ Börje Johansson, ${ }^{1,2}$ \\ Natalia V. Skorodumova, ${ }^{2}$ and Adri C. T. van Duin ${ }^{3}$ \\ ${ }^{1}$ Applied Materials Physics, Department of Materials Science and Engineering, The Royal Institute \\ of Technology, SE-100 44 Stockholm, Sweden \\ ${ }^{2}$ Condensed Matter Theory Group, Department of Physics, Uppsala University, Box 530, \\ SE-751 21 Uppsala, Sweden \\ ${ }^{3}$ Materials and Process Simulation Center (139-74), California Institute of Technology, Pasadena, \\ California 91125, USA
}

(Received 18 April 2008; accepted 15 October 2008; published online 19 November 2008)

The melting curve of hydrogen was computed for pressures up to $200 \mathrm{GPa}$, using molecular dynamics. The inter- and intramolecular interactions were described by the reactive force field (ReaxFF) model. The model describes the pressure-volume equation of state solid hydrogen in good agreement with experiment up to pressures over $150 \mathrm{GPa}$, however the corresponding equation of state for liquid deviates considerably from density functional theory calculations. Due to this, the computed melting curve, although shares most of the known features, yields considerably lower melting temperatures compared to extrapolations of the available diamond anvil cell data. This failure of the ReaxFF model, which can reproduce many physical and chemical properties (including chemical reactions in hydrocarbons) of solid hydrogen, hints at an important change in the mechanism of interaction of hydrogen molecules in the liquid state. (C) 2008 American Institute of Physics. [DOI: 10.1063/1.3013704]

\section{INTRODUCTION}

Most materials have a melting temperature that is monotonically increasing with pressure, i.e., $d T / d P$ is positive. However, there are a few cases, such as water, where this condition does not hold, due either to open crystal structures or changes in the electronic structure when compressing. In these cases the liquid phase is found to be denser than the solid phase.

There has been a discussion about the existence of a maximum in the melting curve of hydrogen at high pressures. Bonev et al. ${ }^{1}$ in 2004 showed that this maximum can indeed be obtained from two-phase ab initio simulations and proposed a gradual softening of the intermolecular repulsive interactions as its cause.

More recently, Pickard and Needs ${ }^{2}$ performed density functional theory (DFT) simulations of high-pressure $\mathrm{H}_{2}$ to obtain its zero-temperature phase diagram. This diagram seems to confirm that phase I of solid $\mathrm{H}_{2}$, consisting of randomly oriented molecules located at sites in a hexagonal close packed (HCP) crystal structure, is not stable above $110 \mathrm{GPa}$ and undergoes a transition to phase II and then to phase III (stable above $150 \mathrm{GPa}$ ). These phases preserve the $\mathrm{HCP}$ arrangement of the molecules, but their orientations are increasingly ordered.

Accurate simulation of the melting process involves large system sizes and, at the same time, averaging over a large number of statistically independent configurations. This is feasible in molecular dynamics using fully empirical interatomic potentials, but at a cost of a simplified model for interactions. As an earlier attempt to bypass this compromise

a)Electronic mail: idavis@kth.se. between computation time and accuracy of the model, largescale molecular dynamics simulations of shockwave propagation in liquid deuterium were performed by Belonoshko et $a l .{ }^{3}$ These simulations involved extremely high pressures and used a refined intermolecular potential adjusted from empirical and $a b$ initio data.

In this work we perform molecular dynamics simulations in order to calculate the melting curve for hydrogen up to $200 \mathrm{GPa}$. Our simulations are based on an empirical force field model developed by van Duin et al., ${ }^{4}$ which is accurate enough to reproduce many properties and chemical reactions of hydrocarbons. While the system sizes and simulation times needed for simulating the melting process can be prohibitive in pure $a b$ initio methods, the use of empirical force fields like this allows us to maintain a reasonable accuracy in the modeling of the complex interactions of the hydrogen atoms in affordable computer time.

\section{METHOD}

To determine the melting curve, we applied the procedure devised by Belonoshko et al., ${ }^{5}$ which we will refer to as the Z-method. According to this method, a system in the high-pressure limit being simulated in the microcanonical $(N V E)$ ensemble at a temperature corresponding to its limit of superheating $\left(T_{\mathrm{LS}}\right)$ will "drop" naturally to the melting temperature $T_{m}$ for a given pressure. By performing long microcanonical simulations at different cell volumes (matching the different pressure ranges we want to access), it is possible to obtain points directly on the melting curve without any external intervention on the dynamics of the melting process (such as equilibrating the temperature via "thermostat" algorithms). 
The preparation of the starting solid structures was as follows: we considered a $\mathrm{HCP}$ crystal consisting of $250 \mathrm{H}_{2}$ molecules as the starting structure. The orientations of the molecules were set initially at random, and then the system as a whole was thermalized at $T=50 \mathrm{~K}$ using molecular dynamics with simple rescaling of velocities for $2.5 \mathrm{ps}$ in order to ensure its stability (removing the excess configurational energy it may have). This procedure was repeated for five different densities in order to sample the melting curve at five different pressures.

The intramolecular and intermolecular forces were calculated according to the reactive force field (ReaxFF) model, a reactive force field developed by van Duin et al. ${ }^{4}$ This model has been designed and adjusted to accurately reproduce the chemical bonds in conjugated, nonconjugated, and radical-containing hydrocarbons in terms of geometry (bond lengths and angles) and dissociation curves. It also has the remarkable advantage of being much faster than traditional quantum chemistry (such as DFT) or semiempirical [such as parametrized model number 3 (PM3)] approaches.

Given the quality of the model, we believe ReaxFF is accurate enough to reproduce the behavior of solid and liquid hydrogen at extremely high pressures, where the intermolecular interactions may become really important. The use of this force field makes it possible to perform realistic simulations with hundreds or even thousands of atoms and times of the order of dozens of picoseconds, a scale that is prohibitive in purely $a b$ initio approaches such as DFT or other quantum chemistry schemes.

For each density the system was simulated in the $N V E$ ensemble for 75000 steps, corresponding to $17.5 \mathrm{ps}$ (with a time step of $0.25 \mathrm{fs}$ ) at different initial temperatures in order to construct an isochoric curve $P$ versus $T$. The system evolved freely without any temperature control, and in each case, after reaching the critical superheating temperature $T_{\mathrm{LS}}$, the isochore line dropped to a point $\left(P, T_{m}\right)$ that should fall precisely along the melting curve.

\section{RESULTS}

In order to check the overall reliability of our model for solid hydrogen for a wide range of pressures, we performed simulations to obtain the equation of state $V(P)$ for a temperature $T=300 \mathrm{~K}$. The points obtained (molar volume for a given pressure) are shown in Fig. 1, in really good agreement with experimental data from the work of Loubeyre et al. ${ }^{6}$

The isochore plots for each density, obtained with the Z-method, are shown in Fig. 2, and the melting points extracted from these data (the ones marked with diamonds on each plot) are shown in Table I with their respective error estimations.

In order to make sure that we indeed have a solid behavior at $T_{\mathrm{LS}}$ and a liquid behavior at $T_{m}$ for each of the volumes considered, we compared the mean square displacement (MSD) as a function of time for the molecules (i.e., considering the displacement of the center of mass coordinate of each diatomic molecule) at the critical superheating temperature $T_{\mathrm{LS}}$ (where we still expect to have a solidlike diffusion) with the one at the melting temperature $T_{m}$ (where liquidlike

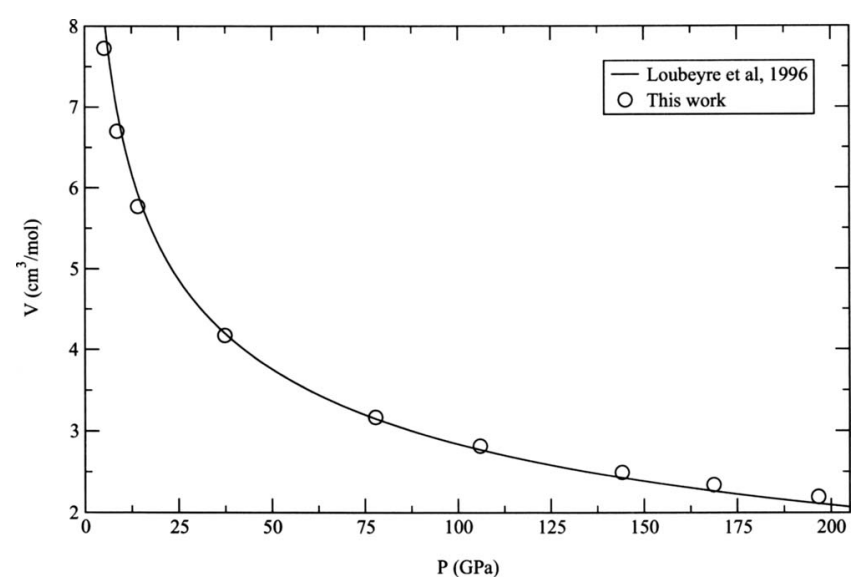

FIG. 1. Equation of state for solid $\mathrm{H}_{2}$ at $T=300 \mathrm{~K}$ obtained using the ReaxFF potential (circles in the figure), compared with experimental data from Loubeyre et al. (Ref. 6) (solid line).

diffusion is expected). As an example of this, Fig. 3 shows the MSD up to $2.5 \mathrm{ps}$ for $P=40 \mathrm{GPa}$ at $T_{\mathrm{LS}}=392 \mathrm{~K}$ and $T_{m}=369 \mathrm{~K}$. For comparison purposes, the MSD at $T$ $=120 \mathrm{~K}$ (solid) and $T=710 \mathrm{~K}$ (liquid) are also included. Here it can be seen that the difference in diffusion is clear: the molecules at $T_{m}$ have a MSD that is linearly increasing for large times, as predicted for liquids (compare with MSD at $T=710 \mathrm{~K}$ ), while at $T_{\mathrm{LS}}$ the displacement reaches a more or less constant value, as molecules in a solid cannot diffuse away from their equilibrium positions in the crystal (compare with MSD at $T=120 \mathrm{~K}$ ).

Figure 4 shows the diffusion coefficient $D$, estimated using the MSD up to $t_{0}=0.62 \mathrm{ps}$ according to

$$
D=\frac{\left\langle r\left(t_{0}\right)^{2}\right\rangle}{6 t_{0}},
$$

for every pressure point in our calculated melting curve. Here we can confirm that for all the pressure range considered, there is a clear differentiation between the diffusion at the critical superheating temperature and at the melting temperature. We can also note that, in general, diffusion decreases with pressure, as expected.

To complete the characterization of the solid and liquid states obtained, we calculated the center of mass radial distribution function $g(r)$ for the $\mathrm{H}_{2}$ molecules. Figure 5 shows $g(r)$ for $P=200.85 \mathrm{GPa}$ at low temperature $(T=120 \mathrm{~K})$, at high temperature $(T=710 \mathrm{~K})$, and at the corresponding melting temperature $(T=443 \mathrm{~K})$ and superheating temperature $(T=463 \mathrm{~K})$. Here it can be seen that the structure differences are noticeable mostly for the intermediate and long ranges.

TABLE I. Melting temperatures for $\mathrm{H}_{2}$ as a function of pressure.

\begin{tabular}{cc}
\hline $\begin{array}{c}\text { Pressure } \\
(\mathrm{GPa})\end{array}$ & $\begin{array}{c}\text { Melting temperature } \\
(\mathrm{K})\end{array}$ \\
\hline $5.65 \pm 0.21$ & $224.30 \pm 4.85$ \\
$37.85 \pm 0.26$ & $368.32 \pm 9.83$ \\
$79.40 \pm 0.61$ & $429.76 \pm 10.59$ \\
$145.59 \pm 0.72$ & $435.24 \pm 12.52$ \\
$200.85 \pm 0.57$ & $443.40 \pm 11.86$ \\
\hline \hline
\end{tabular}



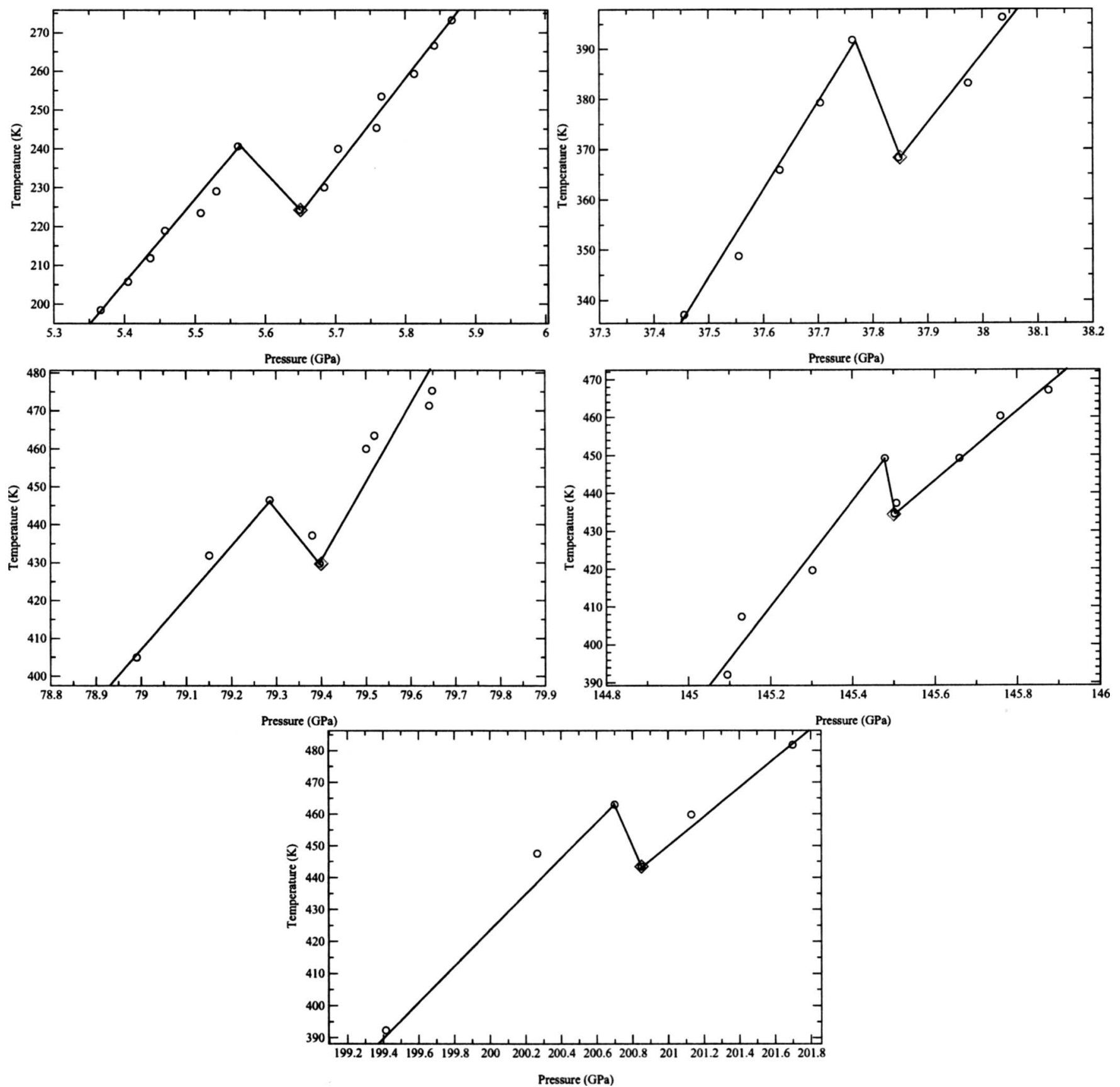

FIG. 2. Isochoric curves for $\rho=0.26,0.48,0.63,0.80$, and $0.91 \mathrm{~g} / \mathrm{cm}^{3}$, close to the respective melting points (marked as diamonds in the different plots). Solid lines are just a guide for the eye.

For instance, some of the structure of the peak at around $4 \AA$ for low-temperature solid "survives" up to the superheating temperature, but it is definitively lost at the melting temperature.

In order to obtain a continuous melting curve, the points obtained were fitted to a Kechin melt equation ${ }^{7}$ as follows:

$$
T_{m}(P)=T_{0}(1+P / a)^{b} \exp (-c P)
$$

where $P$ is the applied pressure, $T_{m}(P)$ the melting temperature corresponding to that pressure, and the values of the four adjustable parameters are $T_{0}=13.884 \mathrm{~K}$ (which corresponds to the melting temperature at zero pressure), $a$ $=0.00042279 \mathrm{GPa}, b=0.29427$, and $c=0.0019589 \mathrm{GPa}^{-1}$. The resulting curve is shown in Fig. 6, in comparison with extrapolations of experimental data and data from $a b$ initio simulations.

The melting temperature for pressures below $5 \mathrm{GPa}$ is in good agreement with existing data (our extrapolated zeropressure melting temperature, $T_{m}=13.884 \mathrm{~K}$, lies within $1 \%$ of the experimental value, $14.01 \mathrm{~K}$ ), but as soon as one moves beyond 4 or $5 \mathrm{GPa}$, our curve becomes flat very quickly. We can see, however, that the asymptotic value of our melting curve $(P$ over $200 \mathrm{GPa})$ agrees again with the existing data.

This fact somehow suggests that there is some component missing in the ReaxFF model of the intermolecular interactions, which becomes dominant in the range of pressures between 5 and $200 \mathrm{GPa}$. As the behavior of the solid 


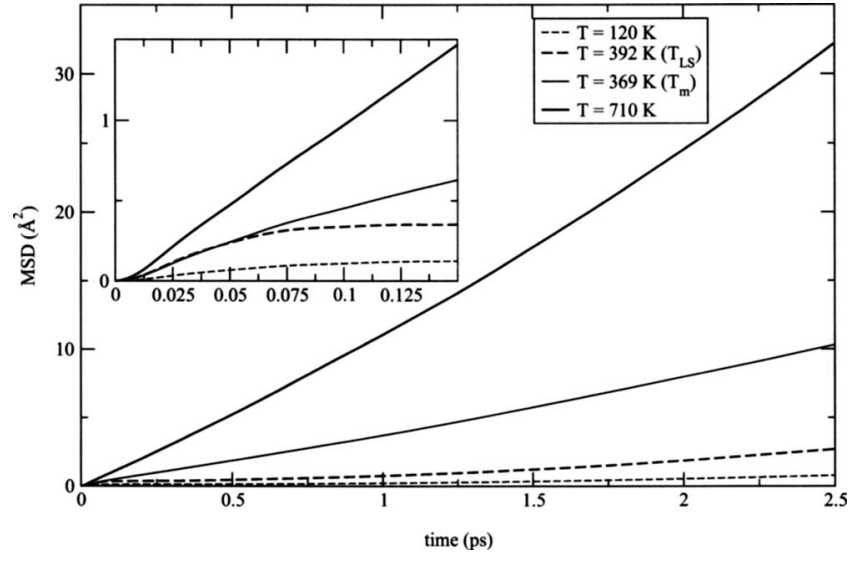

FIG. 3. MSD of $\mathrm{H}_{2}$ molecules for $P=40 \mathrm{GPa}$ at $T=120 \mathrm{~K}$ (thin dashed line), $T_{\mathrm{LS}}=392 \mathrm{~K}$ (thick dashed line), $T_{m}=369 \mathrm{~K}$ (thin solid line), and $T$ $=710 \mathrm{~K}$ (thick solid line). The inset shows the same curves up to $0.15 \mathrm{ps}$.

seems to be well reproduced by ReaxFF at all pressures (Fig. 1 ), we can only conclude that the problem is the description of the liquid state.

To gain some insight on the origin of this, we computed the pressure for the liquid state along the melting curve according to the ReaxFF model and compared it with DFT calculations using the VASP computer code. ${ }^{8}$ The results are shown in Fig. 7.

The origin of the low melting temperatures obtained is then clear: ReaxFF calculations will not reproduce the correct volume differences $\Delta V$ between solid and liquid for pressures between 5 and $200 \mathrm{GPa}$, and therefore the slope of the melting curve,

$$
\frac{d T}{d P}=\frac{\Delta V}{\Delta H},
$$

according to the Clausius-Clapeyron relation, will not be the correct one within this interval.

Despite the low values of $T_{m}$, we can still obtain useful information from the shape of the melting curve. It predicts a slight maximum at a pressure $P=b / c-a=150.22 \mathrm{GPa}$, which is consistent with the experimental data provided by Datchi et al. ${ }^{9}(128 \mathrm{GPa})$ and the ab initio melting curve by Bonev et al. ${ }^{1}$ (82 GPa).

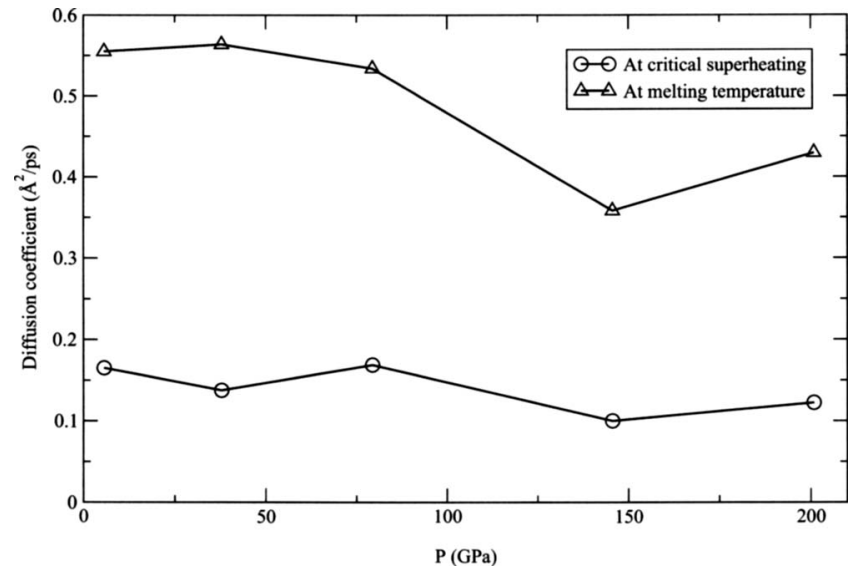

FIG. 4. Diffusion coefficient for $\mathrm{H}_{2}$ molecules compared for each pressure at the melting point (triangles) and superheating point (circles).

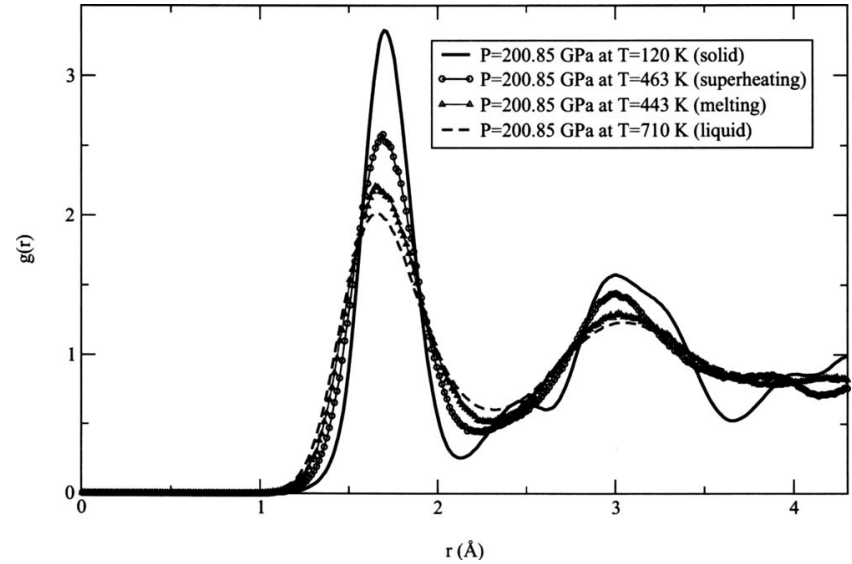

FIG. 5. Center of mass radial distribution function $g(r)$ of $\mathrm{H}_{2}$ molecules at $P=200.85 \mathrm{GPa}$, for low, melting, superheating, and high temperatures.

\section{CONCLUDING REMARKS}

We have computed the melting curve of hydrogen up to $200 \mathrm{GPa}$ according to the ReaxFF model. The curve changes slope at around $150 \mathrm{GPa}$, which seems consistent with existent extrapolations of experimental data and ab initio simulations. Thus the combination of a single-phase method (the Z-method) with a force field interatomic potential is enough to reproduce the existence of a maximum melting temperature in the case of $\mathrm{H}_{2}$.

We noticed, however, that the ReaxFF model fails to reproduce the correct values for pressure in the liquid state, and this causes our melting temperatures to be lower than those reported. The asymptotic value of the melting temperature $(P$ above $200 \mathrm{GPa})$ is, however, well reproduced.

The fact that the ReaxFF model in the case of hydrogen seems to be not transferable between solid and liquid at the same pressure is a very interesting one and, in our opinion, should not be overlooked. In other materials, for example, simple metals like $\mathrm{Fe}$ in the same range of pressures, a

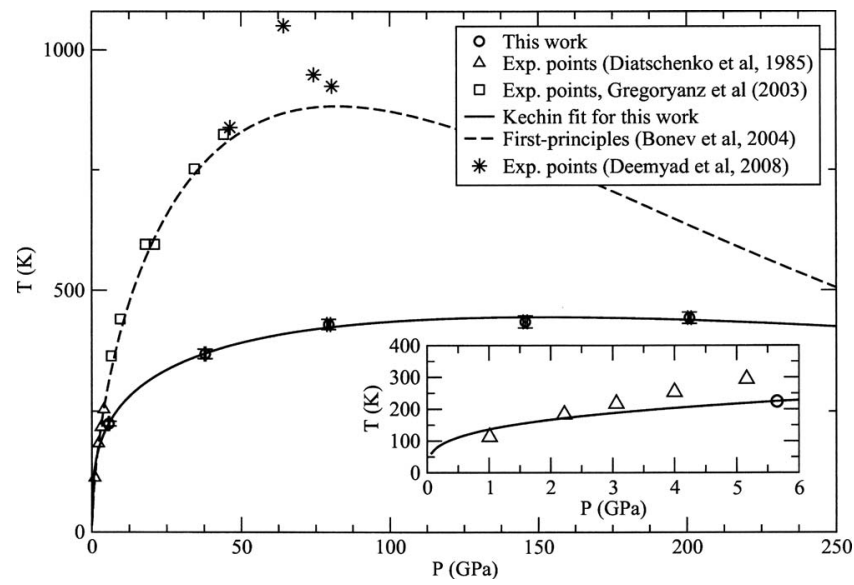

FIG. 6. Melting curve for hydrogen up to $200 \mathrm{GPa}$. The circles represent the individual points obtained with the Z-method and the continuous black line is the Kechin melt equation fit of these points. The triangles are some experimental points from Diatschenko et al. (Ref. 11) up to $5 \mathrm{GPa}$, squares are experimental points from Gregoryanz et al. (Ref. 12) up to $44 \mathrm{GPa}$, and the stars are recent experimental points from Deemyad and Silvera (Ref. 13). The dashed line is the Kechin melt equation according to the $a b$ initio data by Bonev et al. (Ref. 1). 


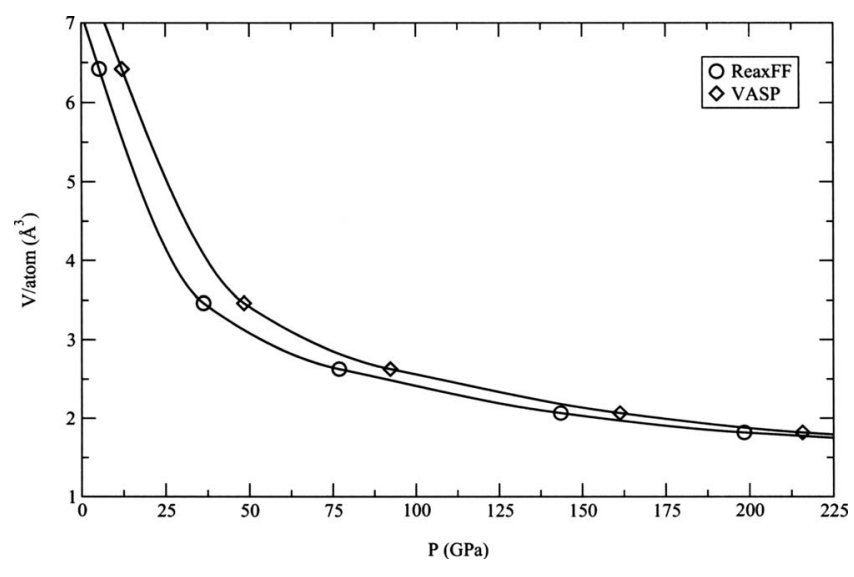

FIG. 7. Equation of state for liquid $\mathrm{H}_{2}$ at $T=0 \mathrm{~K}$ obtained using the ReaxFF potential (marked as circles in the figure), compared with data from DFT calculations (marked as diamonds). The solid lines are "spline" interpolations of these points, drawn as a guide for the eye.

simple embedded-atom interatomic potential can reproduce both solid and liquid behaviors and the correct melting temperatures. ${ }^{10}$ The failure in this concrete case suggests a qualitative change in the nature of the intermolecular interactions in hydrogen when going from a high-pressure solid to a high-pressure liquid.

In principle, it is possible to reparametrize the ReaxFF model by including these new, ab initio data for the high- pressure liquid, as obtained with DFT. This procedure leads to a clear and robust perspective for improvement of the model.

\section{ACKNOWLEDGMENTS}

Computations were performed at the National Supercomputing Center in Linköping and the Parallel Computer Center in Stockholm.

${ }^{1}$ S. A. Bonev, E. Schwegler, T. Ogitsu, and G. Galli, Nature (London) 431, 669 (2004).

${ }^{2}$ C. J. Pickard and R. J. Needs, Nat. Phys. 3, 473 (2007).

${ }^{3}$ A. B. Belonoshko, A. Rosengren, N. V. Skorodumova, S. Bastea, and B. Johansson, J. Chem. Phys. 122, 124503 (2005).

${ }^{4}$ A. C. T. van Duin, S. Dasgupta, F. Lorant, and W. A. Goddard, J. Phys. Chem. A 105, 9396 (2001).

${ }^{5}$ A. B. Belonoshko, N. V. Skorodumova, A. Rosengren, and B. Johansson, Phys. Rev. B 73, 012201 (2006).

${ }^{6}$ P. Loubeyre, R. LeToullec, D. Hausermann, M. Hanfland, R. J. Hemley, H. K. Mao, and L. W. Finger, Nature (London) 383, 702 (1996).

${ }^{7}$ V. V. Kechin, Phys. Rev. B 65, 052102 (2001).

${ }^{8}$ G. Kresse and J. Furthmüller, Comput. Mater. Sci. 6, 15 (1996).

${ }^{9}$ F. Datchi, P. Loubeyre, and R. LeToullec, Phys. Rev. B 61, 6535 (2000).

${ }^{10}$ A. B. Belonoshko, R. Ahuja, and B. Johansson, Phys. Rev. Lett. 84, 3638 (2000).

${ }^{11}$ V. Diatschenko, C. W. Chu, D. H. Liebenberg, D. A. Young, M. Ross, and R. L. Mills, Phys. Rev. B 32, 381 (1985).

${ }^{12}$ E. Gregoryanz, A. F. Goncharov, K. Matsuishi, H. Kwang Mao, and R. J. Hemsley, Phys. Rev. Lett. 90, 175701 (2003).

${ }^{13}$ S. Deemyad and I. F. Silvera, Phys. Rev. Lett. 100, 155701 (2008). 